\title{
Towards Feature Fusion - The Synthesis of Contour Sections Distinguishing Contours from Different Classes
}

\author{
Dag Pechtel and Klaus-Dieter Kuhnert \\ University of Siegen \\ 57068 Siegen, Germany \\ \{pechtel, kuhnert\}@pd.et-inf.uni-siegen.de \\ http://www.pd.et-inf.uni-siegen.de
}

\begin{abstract}
In real world problems, where the objects are in general complex and deformed, the automatic generation of local characteristics is necessary in order to distinguish different object classes.

This paper presents an approach towards the automatic synthesis of significant local contour sections of closed, discrete, complex, and deformed 2D-object contours for the distinction of different classes. Neighboring contour points are determined and synthesized (feature fusion) into feature groups. Exclusively with the help of these feature groups the method distinguishes between different 2D-object contour classes of a certain domain. The basic idea is to get only the necessary information of a contour or a contour class for recognition.
\end{abstract}

\section{Introduction}

A basic problem in the field of pattern recognition is the automatic synthesis of elementary features to higher level features (feature fusion), that can distinguish between certain pattern classes of a task domain.

This paper presents an approach for closed discrete 2D-object contours, whose base features, the contour points, are nearly automatically fused to local and significant feature groups, called the significant contour sections. These significant contour sections distinguish a contour from the contours of other contour classes in a task domain. E.g. a significant contour section of a bottle could be its bottle neck and significant contour sections of a fish could be its fins. The method even works with contours that are complex and/or badly deformed.

There are many approaches in literature concerned with the analysis of contour similarities [L97]. There are alignment approaches [U96], the use of invariant properties (basics in [PM47]) that is often a global approach, and the use of parts ([HS97], [LL98b], [KTZ94], [SIK95], [STK95]) that is sometimes based on shape simplification [LL98a], or structural descriptions ([KW96], [P77]). Some works deal with deformation and minimize a cost function: [BCGJ98] define 'elastic energy' needed to transform one contour to the other. [SGWM93] is concerned with shape interpolation, also known as morphing, or the sequence of contour points 
is interpreted as a string in which points are substituted (shifted), inserted, and deleted ([PK00], [SK83]). This work is based on [PK00]. It uses shares of the alignment approach and isn't psychophysically motivated. This work is concentrated on the nearly automatic synthesis of local contour sections for recognition and is a step towards the autonomous creation of abstract local characteristics of a contour class consisting of a variety of similar, but not equal objects (eg. the birds in fig. 8). Besides the variety, we also interpret outer influences (eg. occlusion, bending) to an object, autonomous motions (eg. stretching out an arm) of an object, and different projections of an object all as deformations.

Basic notations used in this article are presented in section 2. In section 3 the determination of important contour sections, which distinguish between two different 2D-object contours, is briefly sketched. Section 4 shows how the important contour sections of one contour are combined to significant contour sections of that contour. In section 5 experimental results are presented based on a data set of deformed contours of reusable material collections (plastic bottles, beverage cartons (fig. 7) and a data set of birds, fishes, and plastic bottles (fig. 8). With a summary in section 6 this paper concludes.

\section{Notation}

The task domain $D$ consists of $N$ classes $K_{i}$ :

$$
D=\left\{K_{1}, \ldots, K_{i}, \ldots, K_{N}\right\}
$$

Each class $K_{i}$ consists of $m_{i}$ closed, discrete 2D-object contours $C_{i, j}$ :

$$
K_{i}=\left\{C_{i, 1}, \ldots, C_{i, j}, \ldots, C_{i, m_{i}}\right\} .
$$

Each contour $C_{i, j}$ consists of $n_{i, j}$ neighboring successive points $P_{i, j, k}$ with equal Euclidean distances $d$. Each contour consists also of $n_{i, j}$ sections $\tilde{C}_{i, j, k}^{l_{i, j, k}}$ :

$$
\begin{gathered}
C_{i, j}=\left\{P_{i, j, 1}, \ldots, P_{i, j, k}, \ldots, P_{i, j, n_{i, j}}\right\} \\
C_{i, j}=\left\{\tilde{C}_{i, j, 1}^{l_{i, j, 1}}, \ldots, \tilde{C}_{i, j, k}^{l_{i, j, k}}, \ldots, \tilde{C}_{i, j, n_{i, j}}^{l_{i, j, n_{i, j}}}\right\} .
\end{gathered}
$$

Each section $\tilde{C}_{i, j, k}^{l_{i, j, k}}$ consists of $\left(2 l_{i, j, k}+1\right), l_{i, j, k} \geq 1$ points $P_{i, j, k}$ :

$$
\tilde{C}_{i, j, k}^{l_{i, j, k}}=\left\{P_{i, j, k-l_{i, j, k}}, \ldots, P_{i, j, k}, \ldots, P_{i, j, k+l_{i, j, k}}\right\} .
$$

Therefore, neighboring sections have at least 2 common points $P_{i, j, k}$. Finally each point $P_{i, j, k}$ consists of a x- and a y-coordinate:

$$
P_{i, j, k}=\left\{x_{i, j, k}, y_{i, j, k}\right\} .
$$

Each section $\tilde{C}_{i, j, k}^{l_{i, j, k}}$ has an orientation vector $o_{i, j, k}$ :

$$
o_{i, j, k}=P_{i, j, k+l_{i, j, k}}-P_{i, j, k-l_{i, j, k}} .
$$




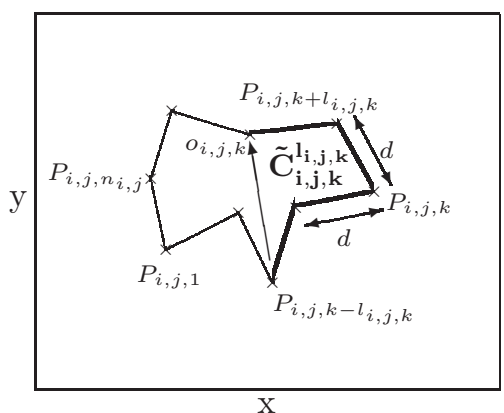

Fig. 1. Example of a closed discrete contour $C_{i, j}$ with one of $n_{i, j}$ sections $\tilde{C}_{i, j, k}^{l_{i, j, k}}$ (marked bold; $l_{i, j, k}=2$ ) and the corresponding orientation vector $o_{i, j, k}$. Successive points have all equal Euclidean distances $d$

An example of such a discrete contour is shown in fig.1.

Two contours are distinguished with the following indices: $C_{i, j}$ and $C_{r, s}$. Two points or sections are distinguished as follows: $P_{i, j, k}, P_{r, s, t}$ and $\tilde{C}_{i, j, k}^{l_{i, j, k}}, \tilde{C}_{r, s, t}^{l_{r, s, t}}$, respectively.

A local comparison of two sections results in a local similarity $\lambda_{(i, j, k),(r, s, t)}$ :

$$
f_{1}: \quad \lambda_{(i, j, k),(r, s, t)}=f_{1}\left(\tilde{C}_{i, j, k}^{l_{i, j, k}}, \tilde{C}_{r, s, t}^{l_{r, s, t}}\right)
$$

and is entered in the $k$ th row and in the $t$ th column of a local similarity matrix $\Lambda^{(i, j),(r, s)}$ :

$$
\Lambda_{k, t}^{(i, j),(r, s)}=\lambda_{(i, j, k),(r, s, t)}
$$

which results from a local similarity analysis of all possible section pairs of two contours $C_{i, j}$ and $C_{r, s}$ :

$$
f_{2}: \quad \Lambda^{(i, j),(r, s)}=f_{2}\left(C_{i, j}, C_{r, s}\right) .
$$

The point indices have to be calculated modulo, because the contours are closed.

\section{Determination of Important Contour Sections}

In [PK00] a method is presented for the determination of important contour sections that distinguish between 2 different contours. That method is based on a local similarity analysis of all possible section pairs $\tilde{C}_{i, j, k}^{l_{i, j, k}}, \tilde{C}_{r, s, t}^{l_{r, s, t}}$ with a constant number of points $\left(2 l_{i, j, k}+1\right)=\left(2 l_{r, s, t}+1\right)=$ const and is shortly recapitulated here in a little changed form.

In fig. 2 is shown an example for the local comparison of two sections $\tilde{C}_{i, j, k}^{2}$, $\tilde{C}_{r, s, t}^{2}$ with $l_{i, j, k}=l_{r, s, t}=2$. First, the sections were translated, that their starting points $P_{i, j, k-2}, P_{r, s, t-2}$ coincided with the origin of the Carthesian coordinate system. Second, the sections were rotated around the origin that their orientation vectors $o_{i, j, k}, o_{r, s, t}$ coincide with the positive x-axis. And third, section $\tilde{C}_{r, s, t}^{2}$ 


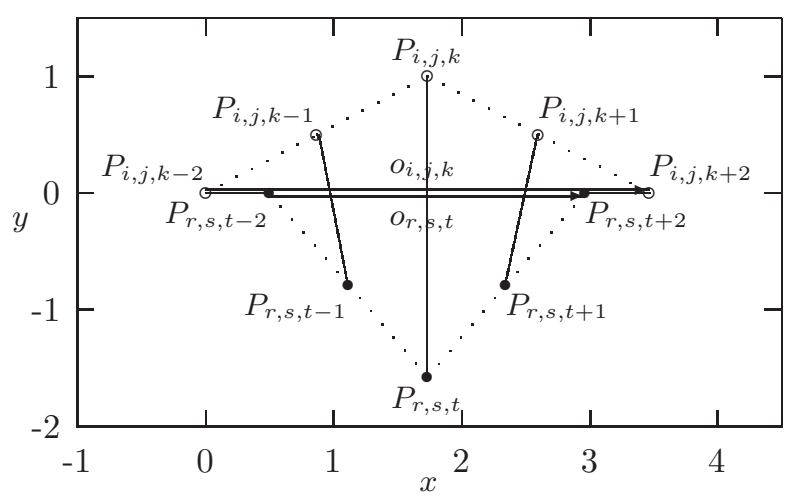

Fig. 2. Example for the comparison of two local contour sections $\tilde{C}_{i, j, k}^{2}, \tilde{C}_{r, s, t}^{2}$ with $l_{i, j, k}=l_{r, s, t}=2$ after translation, rotation (orientation vectors $o_{i, j, k}, o_{r, s, t}$ coincide with the positive $x$ ), and minimizing the sum of Euclidean distances of relating contour points. The 5 relating contour points are connected with 5 straight lines

was translated along the $x$-axis, that the sum of squared Euclidean distances of relating contour points, eg. starting points or ending points of the sections, is minimized. The 5 relating contour points are connected with 5 straight lines in fig. 2 .

Here, we search for the maximum number of points $l_{i, j, k}=l_{r, s, t}$ of the section pair $\tilde{C}_{i, j, k}^{l_{i, j, k}}, \tilde{C}_{r, s, t}^{l_{r, s, t}}$ around the points $P_{i, j, k}, P_{r, s, t}$, that the local distance (dissimilarity) $\epsilon \leq E$. $E$ is the given maximum permitted distance (dissimilarity) between 2 sections. $\epsilon$ is calculated with an approximate one-to-one point matching ([PK00]; a survey can be found in [AG96]).

With this definitions and the notation in section 2, the steps for the calculation of the $\Lambda_{k, t}^{(i, j),(r, s)}$ are combined in the algorithm shown in fig. 3. This results in a $n_{i, j} \times n_{r, s}$ - matrix $\Lambda^{(i, j),(r, s)}=\left[\Lambda_{k, t}^{(i, j),(r, s)}\right]$ of local similarities. The elements $\Lambda_{k, t}^{(i, j),(r, s)}$ give the maximum length of the sections $\tilde{C}_{i, j, k}^{l_{i, j, k}}$ and $\tilde{C}_{r, s, t}^{l_{r, s, t}}$ around the points $P_{i, j, k}$ and $P_{r, s, t}$, with respect to a given maximum dissimilarity $E$. In other words, $\Lambda_{k, t}^{(i, j),(r, s)}$ gives the osculating length of two contour sections that can be treated equal in respect to this fixed maximum error $E$.

In order to get the transformation that possesses minimum cost for the deformation from $C_{i, j}$ to $C_{r, s}$ exactly one monotone discrete path (list of neighbored elements) $\hat{P}_{\psi^{\min }}^{(i, j),(r, s)}$ is searched in $\Lambda^{(i, j),(r, s)}$. For that, the cost function

$$
G_{(i, j),(r, s)}=\sum_{z=1}^{Z} \hat{P}_{\psi_{z}}^{(i, j),(r, s)}, \forall \psi \in\left\{1,2, \ldots, \psi^{\min }, \ldots, \Psi\right\} \quad \rightarrow \max
$$

with $\max \left(n_{i, j}, n_{r, s}\right) \leq Z<\left(n_{i, j}+n_{r, s}\right)$ is maximized with the help of $d y$ namic programming technique (e.g. [B57], [SK83]). Because of the existence of 


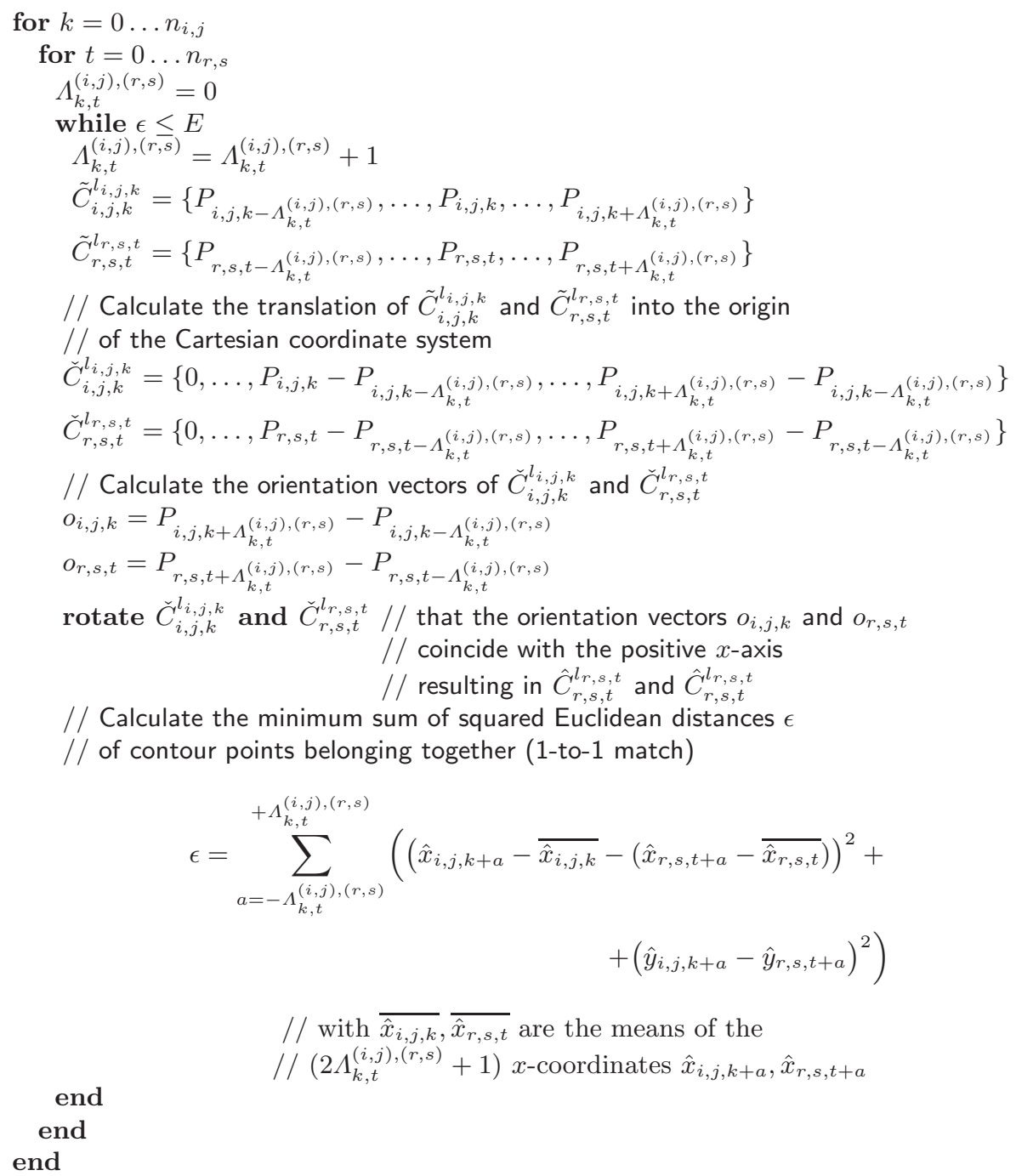

Fig. 3. Algorithm for calculating the elements $\Lambda_{k, t}^{(i, j),(r, s)}$ of the local similarity matrix $\Lambda^{(i, j),(r, s)}$ of 2 contours $C_{i, j}, C_{r, s}$. Comments are marked with //

$\Psi=\min \left(n_{i, j}, n_{r, s}\right)$ possible start elements for $\hat{P}_{\psi}^{(i, j),(r, s)}$ in $\Lambda^{(i, j),(r, s)}$ (rotation), that path is selected over all $\psi$ causing the lowest cost (invariance against rotation).

Sections of $\hat{P}_{\psi \text { min }}^{(i, j),(r, s)}$ that contribute a little to $G_{(i, j),(r, s)}$, i.e. the osculating length is short, map those contour sections of $C_{i, j}$ and $C_{r, s}$ onto each other, that are different. Thus, those contour sections are important contour sections $I$ for the distinction of the contours $C_{i, j}$ and $C_{r, s}$. 

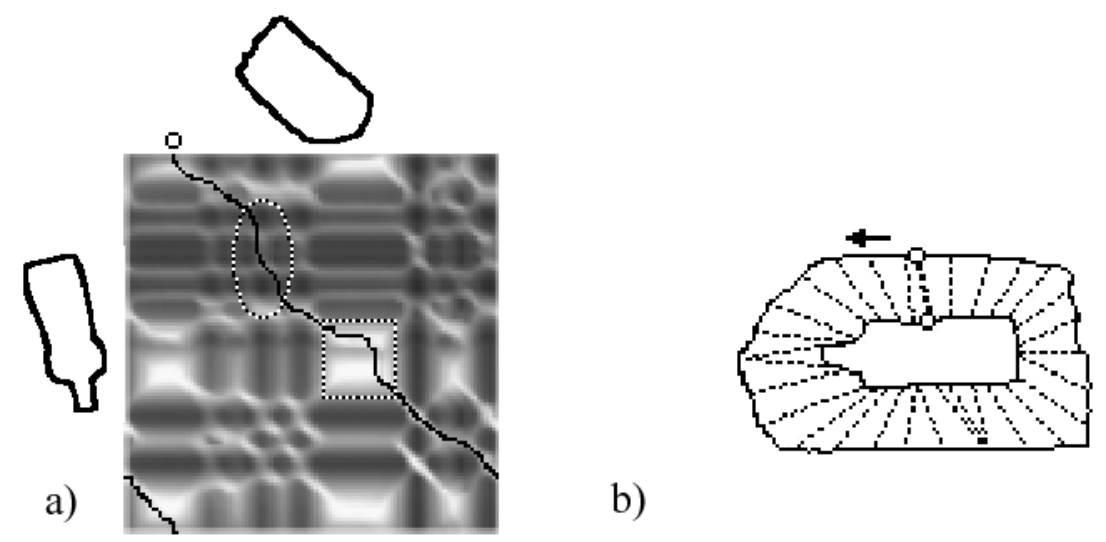

b)

Fig. 4. Mapping of contours $C_{1,5}$ and $C_{2,3}$ (fig. 7) onto each other with lowest cost: a) Visualized similarity matrix $\Lambda^{(1,5),(2,3)}$ of contours $C_{1,5}$ (plastic bottle) and $C_{2,3}$ (beverage carton) with path $\hat{P}_{\psi^{\text {min }}}^{(1,5),(2,3)}$ (marked in black), b) Visualized contour points mapped onto each other with respect to $\hat{P}_{\psi^{\min }}^{(1,5),(2,3)}$ in fig. $4 \mathrm{a}$

An example of such a path $\hat{P}_{\psi^{\text {min }}}^{(1,5),(2,3)}$ for the contours $C_{1,5}$ and $C_{2,3}$ (fig. 7 ) is shown in fig. 4. The local similarity matrix $\Lambda^{(1,5),(2,3)}$ with $\hat{P}_{\psi \min }^{(1,5),(2,3)}$ is shown in fig. 4a. Elements of $\Lambda^{(1,5),(2,3)}$ are scaled between 0 and 255 . Thus, $\Lambda^{(1,5),(2,3)}$ can be visualized as an image. Light (marked with a dotted square) and dark (marked with a dotted ellipse and belongs to the bottle neck) sections respectively in

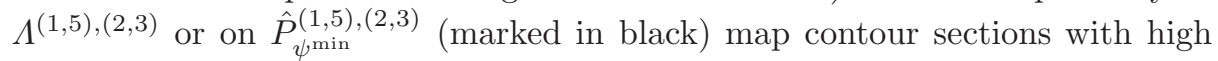
and low local similarity respectively onto each other. $\hat{P}_{\psi^{\min }}^{(1,5),(2,3)}$ starts at an element in the first row marked with a little circle and ends in the last row $\left(\hat{P}_{\psi_{\text {min }}^{(1,5),(2,3)}}\right.$ have to be always "closed" - imagine that the visualized $\Lambda^{(1,5),(2,3)}$ is bent to a torus). Corresponding circles are found in fig. $4 \mathrm{~b}$, too. Here, the mapping of every 20 th contour point is visualized with respect to contour $C_{1,5}$.

For further details see [PK00].

\section{Synthesis of Significant Contour Sections}

With the method described in section 3 important contour sections $I$ can be determined, that distinguish between two 2D-object contours.

This section describes an approach how to generate significant contour sections $S$ for a fixed contour $C_{A, B}$, belonging to a fixed contour class $K_{A}$, from all important sections $I$, that came from the comparison with all contours $C_{r, s}$ of the other classes $K_{r}, r \neq A$. 
First, all local similarity matrices $\Lambda^{(A, B),(r, s)}, A \neq r$ and all paths $\hat{P}_{\psi^{\min }}^{(A, B),(r, s)}$, $A \neq r$ have to be calculated (see section 3). Those sections of $C_{A, B}$, which belong to elements $z$ of $\hat{P}_{\psi^{\text {min }}}^{(A, B),(r, s)}$ satisfying the condition

$$
\hat{P}_{\psi_{z}^{\min }}^{(A, B),(r, s)}<\Theta
$$

are the important contour sections of $C_{A, B} \cdot \Theta$ is constant for all $I$ and is called importance value. I.e., now there is a list of all $I$ of the contour $C_{A, B}$ with respect to all contours $C_{r, s}$. In general, this list includes both $I$, that overlap each other, i.e. they have also equal contour points, and $I$, that do not overlap each other, i.e. they do not have a single contour point in common.

An example in principle is shown in fig. 5. The task domain $D$ consists of 3 classes. Class $K_{1}$ consists of 3 rectangles, class $K_{2}$ consists of 2 ellipses, and class $K_{3}$ consists of 1 triangle. In this example the fixed contour $C_{A, B}=C_{1,2}$. The important contour sections of $C_{1,2}$ and the corresponding important contour sections of all contours of the other classes $K_{2}, K_{3}$ are marked in bold. This results in the marked 4 groups of overlapping important contour sections.

A reasonable assumption is that contours of the same class are more similar to themselves than contours of different classes. Another reasonable assumption is that contours of the same class are more similar to themselves because they have significant contour sections $S$. The last assumption is reasonable because human beings are able to recognize objects even if the objects are occluded. And there is another assumption: many overlapping important contour sections $I$ with only a little variation in their position on the contour give a hint that this sections $I$ are significant contour sections $S$ of this contour with respect to the task domain $D$. But which $I$ is the representative one? There is no general answer to this question. If there are $Q_{p}$ overlapping important sections $I_{q}, 1 \leq q \leq Q_{p}$ a better way is to answer the follwing question:

How are $Q_{p}$ overlapping $I_{q}$ combined to $1 S_{p}$ ?

There are many possible approaches to do that. One possibility is to take the minimum starting point index $\check{s}_{q}^{\min }=\min \left\{\check{s}_{q}\right\}$ and the maximum ending point index $\check{e}_{q}^{\max }=\max \left\{\check{e}_{q}\right\}$ as starting and ending point indexes $\check{S}_{p}, \check{E}_{p}$ of $S_{p}$. But this results in very long $S_{p}$. Good results were obtained with the calculation of the arithmetic means $\check{s}_{q}, \check{e}_{q}$ and the variances $\overline{\check{s}_{q}}, \overline{\check{e}_{q}}$ both of the $Q_{p}$ starting point indexes $\check{s}_{q}$, and the $Q_{p}$ ending point indices $\check{e}_{q}$ of all overlapping $I_{q}$. Then, this results in the following starting and ending point indices $\breve{S}_{p}, \check{E}_{p}$ of the $S_{p}$ :

$$
\check{S}_{p}=\operatorname{round}\left(\check{\check{s}_{q}}-\sqrt{\overline{\overline{\check{s}_{q}}}}\right) \quad, \quad \check{E}_{p}=\operatorname{round}\left(\overline{\check{e}_{q}}+\sqrt{\overline{\overline{\check{e}_{q}}}}\right) \quad, 1 \leq p \leq \Pi \text {. }
$$

$\Pi$ is the total number of $S_{p}$ of a contour and round(...) means: round to the nearest integer. A simple example for the combination (fusion) of two $I_{q}$ is shown in fig. 6.

Since contour $C_{A, B}$ could have several $S_{p}$, another question arises: 


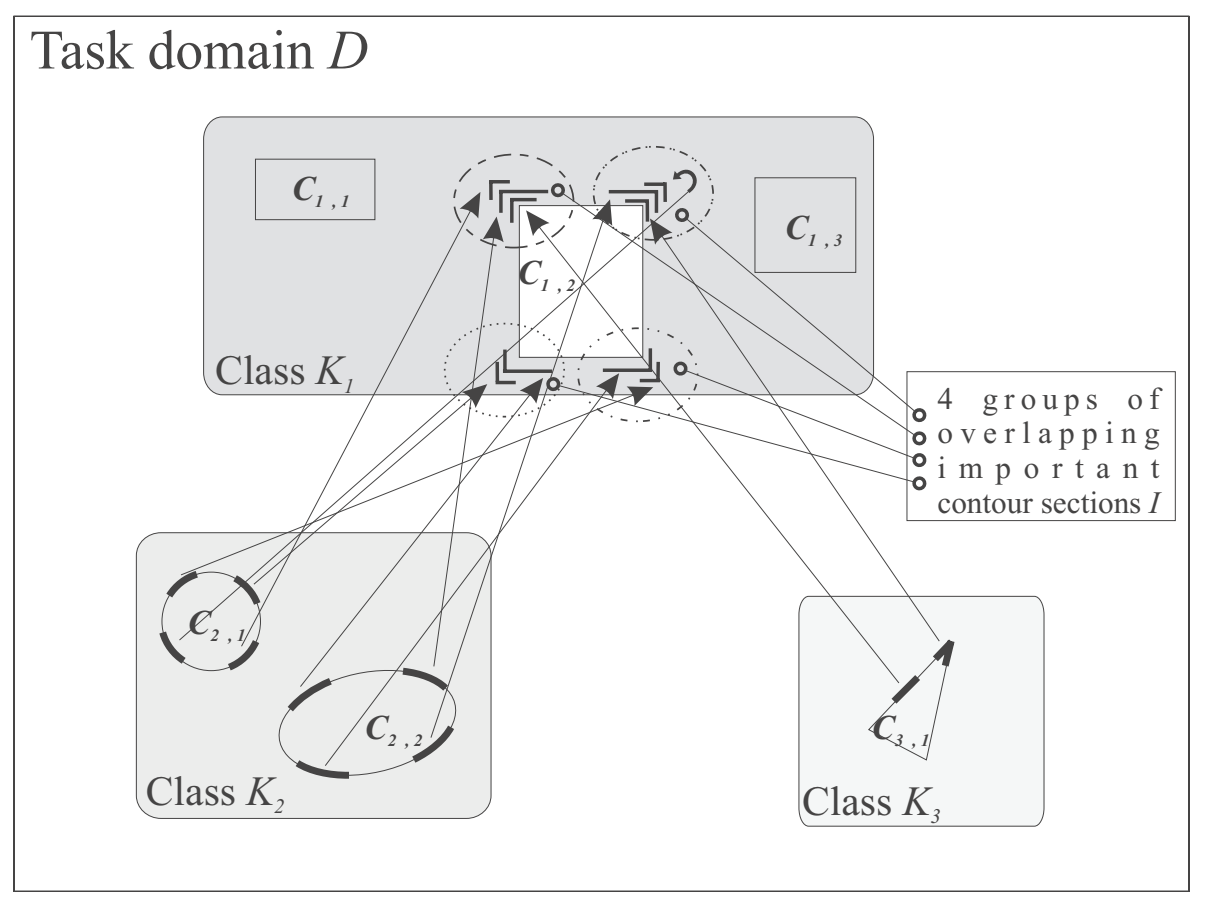

Fig. 5. Example for overlapping and not overlapping important contour sections: 3 classes consisting of 3 rectangles, 2 ellipses, and 1 triangle $\left(K_{1}, K_{2}, K_{3}\right)$ are the task domain. Here, contour $C_{1,2}$ is the fixed contour. All important contour sections of $C_{1,2}$ and all corresponding contour sections of the contours of the other classes $K_{1}, K_{2}$ are marked in bold. This results in 4 different groups each consists of overlapping important contour sections (marked with different dotted ellipses), but no important contour sections of two different groups are overlapping each other

On what depends the significance of the generated $S_{p}$ and what is a reasonable significance measure $\sigma_{p}$ ?

In general the contour $C_{A, B}$ have several significant sections $S_{p}$. It's reasonable to limit the number of $S_{p}$. Because of equation 12 there are $S_{p}$ that comes from not overlapping important sections $I_{q}$ with path elements $\hat{P}_{\psi_{z}^{\min }}^{(A, B),(r, s)}$ near $\Theta$. These $S_{p}$ have only a few points. Therefore a reasonable significance measure $\sigma_{p}$ of $S_{p}$ should have at least 3 dependencies:

- The bigger the number $Q_{p}$ of overlapping $I_{q}$, the bigger the significance of $S_{p}$.

- The smaller the variation

$$
v_{p}=\overline{\overline{s_{q}}}+\overline{\overline{e_{q}}}
$$



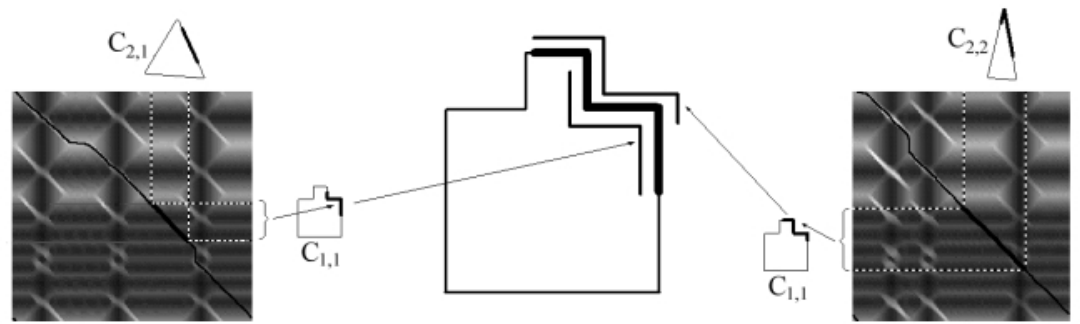

Fig. 6. Example for the fusion of $2 I_{q}$ of a sketched bottle $C_{1,1}$ with respect to a class consisting of 2 triangles $C_{2,1}, C_{2,2}$. Important path sections are marked in black. Corresponding important contour sections $I$ are marked bold on the contours

of the starting and ending point indices of the $Q_{p}$ overlapping $I_{q}$, the bigger the significance of $S_{p}$.

- The bigger the mean of the sum of dissimilarities $d_{p}$

$$
d_{p}=\frac{1}{Q_{p}} \sum_{q=1}^{Q_{p}} \frac{1}{w_{q}} \quad \text { with } \quad w_{q}=\sum_{z=\check{s}_{q}}^{\check{e}_{q}} \hat{P}_{\psi_{z}^{\min }}^{(A, B),(r, s)}
$$

between the $Q_{p}$ individual overlapping $I_{q}$ and the $Q_{p}$ corresponding contour sections of the contours $C_{r, s}$, the bigger the significance. $w_{q}$ is the similarity of two individual corresponding important contour sections of $C_{A, B}$ and $C_{r, s}$.

One possibility to combine this 3 named dependencies in a significance measure is as follows:

$$
\sigma_{p}=\frac{Q_{p}^{\alpha} d_{p}^{\beta}}{1+v_{p}^{\gamma}} \quad, \quad \alpha, \beta, \gamma \in R^{+}
$$

that is abbreviated called significance $\sigma_{p}$ of $S_{p} . \alpha, \beta, \gamma$ are weightings for the 3 dependencies. Now, with the equations (12)-(16) one $S_{p}$ is completely described.

With equation 16 it's possible to limit the number of $S_{p}$ on those $S_{p}$, that satisfy the condition

$$
\sigma_{p} \geq \Phi \sigma_{p}^{\max } \quad, 0.0 \leq \Phi \leq 1.0
$$

$\sigma_{p}^{\max }=\max \left\{\sigma_{p}\right\}$ means the maximum significance of all $S_{p}$ of $C_{A, B}$. Those $S_{p}$, that are more significant than other $S_{p}$ with respect to equation (17) are called more significant contour sections $M_{v}, 1 \leq v \leq V$ and the $S_{p}$ that is the one with the highest significance is called most significant contour section $\hat{M}$.

\section{Experiments}

The presented method was tested with a task domain $D_{1}$ consisting of 2 D-object contours of packagings of reusable plastic material (fig. 7). The collecting process 


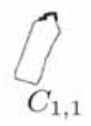<smiles>C1C[As]C1</smiles><smiles></smiles><smiles>C1C[GeH]C1</smiles><smiles>C1C[As]1</smiles><smiles>C1=C[GeH2]1</smiles><smiles>C1C[AsH2]C1</smiles><smiles>C1CCCC1</smiles><smiles>[CH+]1CCC1</smiles><smiles>C1C[GeH2]C1</smiles><smiles>[C]1CCCC1</smiles><smiles>[C]1CCCCC1</smiles>

Fig. 7. Contours of 6 plastic bottles $\left(C_{1,1}-C_{1,6}\right)$ and 6 beverage cartons $\left(C_{2,1}-\right.$ $C_{2,6}$ ) with their most significant contour sections $\hat{M}$ marked in bold

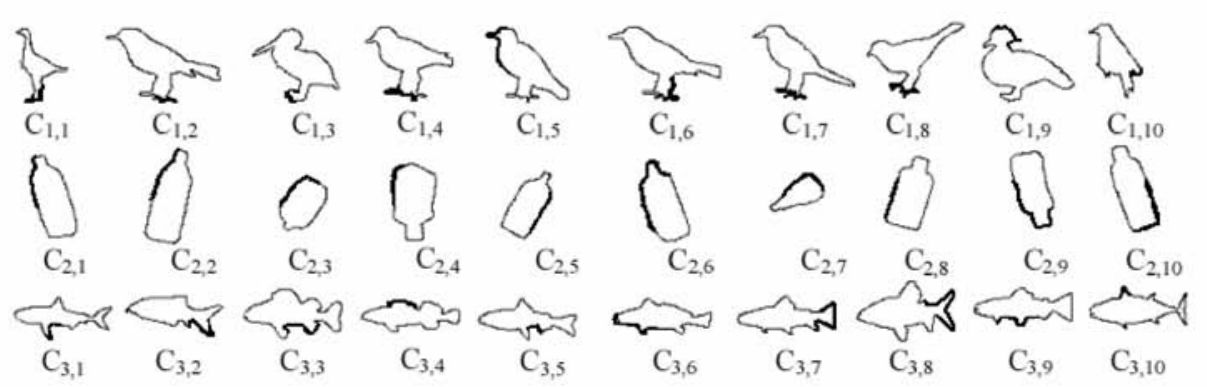

Fig. 8. Contours of 10 birds $\left(C_{1,1}-C_{1,10}\right), 10$ bottles $\left(C_{2,1}-C_{2,10}\right)$, and 10 fishes $\left(C_{3,1}-C_{3,10}\right)$ with their most significant contour sections $\hat{M}$ marked in bold

and the mechanical preparation in a sorting plant make the packagings strongly dirty and deformed. The method was also tested with a more complex task domain $D_{2}$ consisting of 3 classes: fishes, birds and plastic bottles (fig. 8).

All contours are scaled so that their biggest generalized diameters are approximately equal. The contours have from 400 up to 600 contour points and successive contour points have all equal Euclidean distances of 1.2 pixels, because it's a compromise between 1.0 and $\sqrt{2}$ (horizontal and diagonal pixel distance on a square pixel grid). The experiments are carried out with heuristically found parameters. In both cases it seems to be good if the weighting of $Q_{p}$ is high in respect to $d_{p}, v_{p}$.

The results of the experiments are shown in fig. 7 and fig. 8 . The $\hat{M}$ of the contours are marked in bold.

For the bottle class in the task domain $D_{1}$ shown in fig. 7 the most significant contour sections $\hat{M}$ are the bottle necks. Here, it is possible to distinguish this two classes only with the help of the bottle necks. In the more complex task domain $D_{2}$ the $\hat{M}$ of the bottles are not only the bottle necks. Also the sections around one corner are now significant to distinguish the bottles from the birds and the fishes. The most significant sections $\hat{M}$ of the birds are in most cases the claws but there is also a beak. Interesting is bird $C_{1,10}$ which has no claws. 
Here, a tail feather is the $\hat{M}$. The fishes have their most significant sections at their fins. Because of the great variety of the fins the presented method find most significant fins at different positions on the fish contours.

\section{Conclusion}

A method was presented in this paper, that nearly automatically, supervised learns those contour sections $M_{v}$ of a certain contour, with which this contour can be distinguished from contours out of other contour classes. We call this generation of feature groups feature fusion: Base features or feature primitives (contour points) are synthesized to feature groups (are fused to more significant contour sections $M_{v}$ ). Now, the fused more significant contour sections $M_{v}$ can serve as knowledge base of a classifier for assigning an unknown contour to a certain contour class.

Experimental results were made with two different task domains. The method extracted that the class of the plastic bottles can be distinguished from the class of the beverage cartons only with the help of the contour sections we call bottle necks. In the other task domain $D_{2}$ the method extracted that the most significant sections $\hat{M}$ of the birds are the claws and the beaks, the $\hat{M}$ of the fishes are the fins, and the $\hat{M}$ of the bottles are the corners and the bottle necks. This seems a little like a human being would do.

A further combination of the more or most significant contour sections $M_{v}, \hat{M}$ of a certain contour class is desirable in order to create abstract contour sections $\hat{A}$. E.g., combining all $\hat{M}$ at the position of the bottle necks into an $\hat{A}$ "BOTTLE NECK". Thus, a problem still to be solved is the problem of abstraction for the single classes. First experiments with a hierarchical classifier based on the most significant sections $\hat{M}$ were made and give encouraging results.

\section{References}

AG96. Alt, H., Guibas, L. J. : Discrete Geometric Shapes: Matching, Interpolation, and Approximation - A Survey. Technical Reports of FU Berlin (1996), Institut für Informatik u. Mathematik. 521

BCGJ98. Basri, R., Costa, L., Geiger, D., Jacobs, D. : Determining the similarity of deformable shapes. Vision Research 38 (1998), 2365-2385. 518

B57. Bellman, R. : Dynamic Programming. Princeton University Press, Princeton, New Jersey (1957). 521

HS97. Hoffman, D. D., Singh, M. : Salience of visual parts. Cognition 63 (1997), 29-78. 518

KTZ94. Kimia, B. B., Tannenbaum, A. R., Zucker, S. : Shapes, shocks, and deformations 1: The components of shape and the reaction-diffusion space. Int. Journal of Computer vision 15(3) (1995), 189-224. 518

KW96. Kupeev, K., Wolfson, H. : A new method of estimating shape similarity. Pattern Recognition Letters 17 (1996), 873-887. 518

LL98a. Latecki, L. J., Lakämper, R. : Discrete Approach to Curve Evolution. In Levi, P., Ahlers, R.-J., May, F. and Schanz, M. (Edts.): Mustererkennung 1998, 20. DAGM-Symposium, 85-92. 518 
LL98b. Latecki, L. J., Lakämper, R. : Shape decomposition and shape similarity measure. In Levi, P., Ahlers, R.-J., May, F. and Schanz, M. (Edts.): Mustererkennung 1998, 20. DAGM-Symposium, 367-376. 518

L97. Loncaric, S. : A survey of shape analysis techniques. Pattern Recognition 31 (1997), 983-1001. 518

P77. Pavlidis, T. :Structural Pattern Recognition. Springer-Verlag, Berlin (1977). 518

PK00. Pechtel, D., Kuhnert, K.-D. : Generating Automatically Local Feature Groups of Complex and Deformed Objects. Appears in Gaul, W., Decker, R. (eds.): Classification and Information Processing at the Turn of the Millenium. Proc. 23th Ann. Conf. of GfKl, Bielefeld, March 10-12, 1999. Springer, Heidelberg, 2000, 237-244. 519, 520, 521, 523

PM47. Pitts, W., McCulloch W. : How we know universals: the perception of auditory and visual forms. Bulleting of Mathematical Biophysics 9 (1947), 127-147. 518

SGWM93. Sederberg, T., Gao, P., Wang, G., Mu, H. : 2D shape blending: An Intrinsic Solution to the Vertex Path Problem. Computer Graphics 38 (1993) 15-18. 518

SIK95. Siddiqi, K., Kimia, B. : Parts of Visual Form: Computational Aspects. IEEE Transactions On Pattern Anal. And Mach. Intell. 17 (1995) 239-251. 518

STK95. Siddiqi, K., Tresnes, K., and Kimia, B. : Parts of visual form: ecological and psychophysical aspects. In Proc. IAPR's Int. Workshop on Visual Form (1994) . 518

SK83. Sankoff, D., Kruskal, B. K. (Eds.): Time warps, String Edits, and Macromolecules: The Theory and Practice of Sequence Comparison. AddisonWesley, Reading (1983). 519, 521

U96. Ullman, S. : High-level Vision. A Bradford Book, The MIT Press, Cambridge, Massachusetts (1996). 518 